\title{
RELACIÓN ENTRE EL ESTADO AFECTIVO Y EL RENDIMIENTO ACADÉMICO EN LOS ESTUDIANTES DE LA INSTITUCIÓN EDUCATIVA MARISCAL CASTILLA - EL TAMBO - 2009. RELATIONSHIP BETWEEN THE CONCERNED AND ACADEMIC PERFORMANCE OF
STUDENTS FROM THE EDUCATIONAL INSTITUTION Mariscal Castilla - El Tambo - 2009.
} Carmen Samaniego Durand ${ }^{12}$, Edgar Gonzales Santos, Moisés Calle Cáceres Jesús, Ingrid Calle Samaniego,

\section{Facultad de Enfermería}

\section{RESUMEN}

El presente trabajo de investigación se planteó como propósito determinar la relación entre la afectividad y el rendimiento académico en los estudiantes de la institución educativa Mariscal Castilla de El Tambo - Huancayo en el 2009; para ello realizamos un estudio descriptivo correlacional, seleccionamos una muestra de 85 adolescentes del $4^{\circ}$ y $5^{\circ}$ año de educación secundaria a los que se les aplicó una encuesta, encontrándose que el $41.2 \%$ de los adolescentes presentan cierta alteración afectiva que se manifiesta en modificaciones en conducta, en el $42.3 \%$ su problema afectivo se manifiesta con una alteración anímica, el $62.4 \%$ expresa su problema afectivo con alteraciones orgánicas (alteraciones gastrointestinales, cefaleas) y al $72.9 \%$ su problema afectivo le ha generado alteración de sus proceso cognitivos; la alteración afectiva se correlaciona positivamente $(r=0.644)$ pudiendo afirmar que el rendimiento académico puede ser explicado en un $41 \%$ por el estado afectivo del adolescente.

Palabras clave: afectividad, rendimiento académico, adolescente.

\section{ABSTRACT}

This research was the work intended to determine the relationship between emotional health and academic performance among students of El Tambo Mariscal Castilla - Huancayo school in 2009 , to make it a descriptive correlational study, we selected a sample of 85 adolescents in the 4th and 5 th year of secondary education to which they were given a survey found that $41.2 \%$ of adolescents have some alteration affective manifested in changes in behavior, in $42.3 \%$ of its affective problem manifests with impaired soul, $62.4 \%$ expressed affective problem with organic disorders (gastrointestinal disturbances, headache) and $72.9 \%$ of its affective problem has led to alteration of their cognitive processes, emotional disturbance is positively correlated ( $r=0.644$ ) and can say that the academic performance can be explained by $41 \%$ by the teen's emotional state.

Key words: Affectivity, academic yield, adolescent.

\section{INTRODUCCIÓN}

La mayoría de adolescentes que sufren alguna alteración del estado afectivo consultan por primera vez en el nivel primario de atención, sin embargo, frecuentemente este tipo de trastornos no es reconocido por el equipo de salud y, cuando lo son, no siempre son tratados en la forma correcta, con los métodos y conocimientos que se dispone en la actualidad.

Esta situación es muy preocupante, pues cuando estos trastornos no son tratados oportunamente y en buena forma, conduce muchas veces a cuadros recurrentes o de evolución crónica,

\footnotetext{
${ }^{12}$ carmensamaniego96@hotmail.com
} 
incapacidades graves, muertes por suicidio 0 prolongado sufrimiento del adolescente y su familia; pero el primer aspecto de la vida del adolescente que es afectada es su rendimiento académico o laboral.

Los trastornos afectivos, y sus formas "encubiertas" por síntomas físicos o malestares diversos, frecuentemente producen confusión y frustración en el Equipo de Atención Primaria, generan consultas médicas repetidas, exámenes, tratamientos inadecuados 0 interconsultas no resolutivas, que sin mejorar a las personas, elevan los costos de la atención médica, topo ello ha llevado a que estas alteraciones del estado afectivo se hallan incrementado en los últimos años principalmente en la población joven, siendo los más afectados los adolescentes; esto afecta su rendimiento laboral y académico. En Estados Unidos, el riesgo de presentar una alteración del estado afectivo en un adolescente es aproximadamente del $12 \%$, y de este grupo, el riesgo que se vea afectado el rendimiento académico es del $65 \%$.

La prevalencia de la depresión en adolescentes mayor oscila entre el 4,6 y el 7,5\% en varones, y entre el 8,0 y el $13,8 \%$ en mujeres. La prevalencia de la distimia es del 3-4\%.

En todos los países industrializados, la incidencia de depresión en adolescentes, manía, suicidio y trastornos psicóticos del estado de ánimo ha ido aumentando en las generaciones nacidas a partir de 1910. Por razones desconocidas, la tasa de incremento sufrió una elevación brusca en las personas nacidas después de 1940; se trata de un verdadero incremento en la incidencia de los trastornos del estado de ánimo (efecto de cohorte) y no es consecuencia de un mejor reconocimiento. Además de ser más frecuentes, los trastornos del estado de ánimo se están manifestando a edades más tempranas (sobre todo los trastornos bipolares).

El suicidio es un problema obvio de salud pública que complica los trastornos del estado de ánimo con mayor frecuencia que otros trastornos. El riesgo de suicidio a lo largo de la vida en los sujetos con trastorno del estado de ánimo es del $10-15 \%$, y el riesgo de tentativas de suicidio aumentó hasta 41 veces en los adolescentes deprimidos, en comparación con aquellos que tenían otros diagnósticos, en el estudio Epidemiologic Catchment Area (Petronis y cols., 1990). Las mujeres realizan tentativas de suicidio con mayor frecuencia que los varones, pero éstos tienen mayores probabilidades de consumarlo. Sin embargo, en otro estudio, el riesgo excesivo de consumar el suicidio en los varones se explicaba de manera absoluta por una mayor prevalencia de abuso de sustancias en los hombres.

El estudio de Cecilia Sogui (1997) focalizado en un solo distrito limeño resalta la prevalencia de un $32.6 \%$ de depresión en adolescentes, explicando que afectaría fundamentalmente a la población femenina en el periodo del climaterio y a la masculina en la temprana juventud.

Estudios más recientes del Instituto Especializado en Salud Mental "Honorio Delgado-Hideyo Noguchi" (2002) indican un 19\% para depresión en adolescentes en general ( $14.5 \%$ para varones y $23.3 \%$ para mujeres). Sobre los trastornos de depresión, este mismo estudio nos muestra una prevalencia de $25.3 \%$ (20.3\% para varones y $30.1 \%$ para mujeres). Sobre trastornos psicóticos, el estudio muestra una prevalencia del $1 \%(0.7 \%$ masculino y $1.3 \%$ femenino).

El estudio epidemiológico en la sierra peruana Ayacucho, Cajamarca y Huaraz realizado por el INSM "Honorio Delgado-Hideyo Noguchi" muestra una prevalencia de vida de trastornos depresivos del $17 \%$, cifra similar a la ya mencionada, existiendo diferencias entre varones y mujeres: $13.3 \%$ y $20.5 \%$ respectivamente. La prevalencia anual es del $7.4 \%$.

No se han realizado estudios sistemáticos en 
la región Junín referidos al hecho, pero por reportes oficiales de la Dirección regional de salud - Junín se observa un incremento del $20 \%$ en el quinquenio 2005-2009, comparado con el quinquenio anterior en la incidencia de trastornos depresivos en los adolescentes.

Con el propósito de conocer la posible relación entre el estado afectivo de los adolescentes y el rendimiento académico en la institución educativa Mariscal Castilla se formula la siguiente pregunta de investigación: ¿Cuál es la relación entre el estado afectivo y el rendimiento académico en los estudiantes de la institución educativa Mariscal Castilla de El Tambo - Huancayo en el 2009?, con el objetivo general de determinar la relación entre el estado afectivo y el rendimiento académico en los estudiantes de la institución educativa Mariscal Castilla de El Tambo - Huancayo en el 2009.

Los objetivos específicos de la investigación fueron: determinar las características del estado afectivo en los estudiantes de la institución educativa mariscal castilla, determinar el rendimiento académico en los estudiantes de la institución educativa mariscal castilla, establecer la relación del estado afectivo (reflejado en los procesos cognitivos) y el rendimiento académico en los estudiantes de la institución educativa mariscal castilla, establecer la relación del estado afectivo (reflejado en el estado físico) y el rendimiento académico en los estudiantes de la institución educativa mariscal castilla, establecer la relación del estado afectivo (reflejado en los estados anímicos) y el rendimiento académico en los estudiantes de la institución educativa mariscal castilla y establecer la relación del estado afectivo (reflejado en la conducta) y el rendimiento académico en los estudiantes de la institución educativa Mariscal Castilla.

\section{MATERIAL Y MÉTODOS}

Se realizó una investigación de tipo descriptivo correlacional de corte transversal, para establecer las características más importantes de la afectividad y el rendimiento académico de los adolescentes y después determina como afecta el estado afectivo al rendimiento académico, los datos se recolectaron en una sola oportunidad. Esta investigación es de nivel descriptivo por que cuenta con un sólo grupo de estudio y establece una relación de características de ambas variables.

La población de referencia fueron 1200 alumnos matriculados en la Institución Educativa Mariscal Castilla de El Tambo, bajo los siguientes criterios de elegibilidad: alumnos que acepten voluntariamente participar en el estudio, alumnos que se encuentren matriculados en el cuarto y quinto año de educación secundaria, alumnos que vivan en el área metropolitana de Huancayo y alumnos que tengan entre 12 y 16 años. Mientras que los criterios de exclusión fueron: alumnos que se muestren poco colaboradoras con el estudio, alumnos que hayan tenido problemas de comportamiento y alumnos que padezcan algún tipo de problema físico o mental. De los 1200 alumnos luego de aplicar los criterios de elegibilidad y exclusión quedaron 820 .

Al aplicar la fórmula con una desviación estándar de 0.9 , un nivel de confianza del $95,0 \%$ y un error de 0.2 , se obtuvo una muestra de 85 alumnos, los que fueron seleccionados por muestreo por conglomerados biétapico con asignación proporcional.

La técnica para la recolección de datos fue una encuesta y el instrumento un cuestionario estructurado, elaborado en función a los objetivos del estudio; que se aplicó a 80 estudiantes del $4^{\circ}$ y $5^{\circ}$ año de educación secundaria, el cuestionario poseía un código preestablecido para mantener el anonimato de los participantes.

Para que el instrumento sea válido, se efectuó su respectiva validación y confiabilidad. La validación de contenido, constructo y criterio 
se realizó mediante un juicio de expertos, conformado por 05 especialistas en salud mental del adolescente. La confiabilidad se realizó mediante una prueba piloto a 30 adolescentes de la institución educativa "Mariscal Castilla de El Tambo", el cual se midió mediante el coeficiente alfa de Cronbach $\alpha=0,939$.

Se aplicó el formulario establecido en las fechas y horarios previa coordinación con el director de la referida institución educativa, fue en el de turno de mañana, el tiempo promedio para la aplicación del instrumento fue de aproximadamente de 30 minutos por adolescente.

Se informó a los adolescentes sobre el estudio, sus fines y la importancia de su colaboración, asimismo, se le comunicó que su participación debe ser voluntaria, se proporcionó a los adolescentes una hoja de consentimiento informado, se hizo entrega del instrumento para su respectivo llenado, por única vez, considerando los criterios de selección y los principios éticos (beneficencia, no maleficencia, justicia y autonomía) y se recogió el instrumento corroborando su respectivo llenado.

Una vez obtenida la información, se ingresó las respuestas obtenidas de los adolescentes a la computadora, con la finalidad de elaborar una base de datos y realizar el análisis estadístico correspondiente, utilizando el programa Microsoft Office Excel 2007 y el SPSS versión 17. Se implementaron análisis correlaciónales para las diferentes categorías encontradas en ambas variables, se utilizó como nivel de significancia estadística 0,05 para todas las comparaciones y se recurrió a la comparación de medias para muestras independientes y para muestras dependientes.

\section{RESULTADOS Y DISCUSIÓN}

La alteración afectiva más frecuente que se ha podido encontrar en los adolescentes objeto del estudio es el ánimo deprimido (52\%), siendo sus características más frecuentes: el aislamiento (47\%), el encerrarse en sí mismo (32\%), las actividades reducidas al mínimo $(25 \%)$, y los accesos de pesimismo (18\%). En el fondo, estas actitudes evocan la forma de respuesta de la persona al peligro, en particular al de ruptura de los lazos de dependencia, que durante bastantes años (toda la niñez) ha mantenido con su entorno sociofamiliar.

\section{Resultados del estado afectivo}

El instrumento aplicado para valorar el estado afectivo indagado sobre diferentes problemas que indican el grado de afección de la efectividad del adolescente, estos problemas están principalmente vinculados a la depresión en adolescentes; los problemas han sido medidos con una escala cualitativa ordinal que considera:

\begin{tabular}{cc}
\hline $\begin{array}{c}\text { Categoría cualitativa } \\
\text { ordinal }\end{array}$ & $\begin{array}{c}\text { Valor cuantitativo } \\
\text { asignado }\end{array}$ \\
\hline Ausente & 0 \\
Ligero & 1 \\
Moderado & 2 \\
Grave & 3 \\
Incapacitante & 4 \\
\hline
\end{tabular}

Al asignar valoración cuantitativa a las categorías cualitativas ordinales se ha podido llevar la medición de las variables de una escala cualitativa a una escala cuantitativa de razón o proporción, con lo que es posible determinar la media aritmética y la desviación estándar así como aplicar toda la estadística paramétrica en el procesamiento de datos y las pruebas de hipótesis.

Los problemas de fatiga y falta de interés en los estudiantes se aproximan a ligero (0.92), la lentitud de pensamiento y de palabra es ligera (1.14) y la agitación (intranquibilidad) es casi nula (0.24), estos problemas se presentan de manera 
muy heterogénea (desviación estándar promedio $=1,05)$.

Los problemas de Humor deprimido (tristeza, inutilidad) en los estudiantes es casi nulo (0.33), la Sensación de culpabilidad es casi nula $(0.22)$, la Idea de suicidio es casi nula (0.28), la Depresión psíquica es ligera (1.11), la Hipocondría es casi nula (0.32), y el Insight (conciencia de enfermedad) es casi nula (0.32), estos problemas se presentan de manera muy heterogénea (desviación estándar promedio = 0,82).

Los problemas de insomnio en los estudiantes es casi nulo (0.25), la boca seca, flatulencia, diarrea, eructos, retortijones es ligera (1.11), las palpitaciones, cefalalgias tiende a ser ligera (0.89), la pesadez, dorsalgias, mialgias es ligera en la mitad y nula en la otra mitad (0.58), la pérdida de la libido tiende a ser ligera (0.64), y la pérdida de peso tiende a nula $(0.40)$, estos problemas se presentan de manera muy heterogénea (desviación estándar promedio = 1,02).

La perdida de la capacidad memorística es ligera (1.19), los problemas de atención y concentración tienden a ser ligeros (1.11), los problemas para comprender tienden a ser ligeros (0.84), el cansancio mental y aburrimiento tiende a ser ligera (0.60), la falta de claridad de pensamiento tiende a ser nula (0.42), y la sensación de abrumamiento tiende a ser nula $(0.40)$, estos problemas se presentan de manera muy heterogénea (desviación estándar promedio $=1,17)$.

\section{Resultados del rendimiento académico}

Para valorar el rendimiento académico se ha recurrido a los registros de notas de las diferentes asignaturas llevadas por los estudiantes objeto de estudio, las calificaciones que se encuentran en escala vigesimal han sido llevadas a las siguientes categorías:

\begin{tabular}{ccc}
\hline $\begin{array}{c}\text { Calificación en } \\
\text { escala vigesimal }\end{array}$ & $\begin{array}{c}\text { Categoría } \\
\text { cualitativa ordinal }\end{array}$ & $\begin{array}{c}\text { Valor } \\
\text { cuantitativo } \\
\text { asignado }\end{array}$ \\
\hline$(0-4>$ & Deficiente & 0 \\
$(5-8>$ & Malo & 1 \\
$(9-12>$ & Regular & 2 \\
{$[12-16>$} & Bueno & 3 \\
$(16-20)$ & Muy bueno & 4 \\
\hline
\end{tabular}

Al asignar valoración cuantitativa a las categorías cualitativas ordinales se ha podido llevar la medición de las variables de una escala cualitativa a una escala cuantitativa de razón 0 proporción, con lo que es posible determinar la media aritmética y la desviación estándar así como aplicar toda la estadística paramétrica en el procesamiento de datos y las pruebas de hipótesis.

El rendimiento académico en la asignatura de matemática es regular (2.20), en la asignatura de comunicación es regular (2.13), en la asignatura de arte es regular (2.07) y en la asignatura de ingles es regular (2.15), el rendimiento académico es muy heterogéneo (desviación estándar promedio = 1,03).

El rendimiento académico en la asignatura de Formación Ciudadana y Cívica tiende a regular (1.95), en la asignatura de Historia, Geografía y Economía tiende a regular (1.96), en la asignatura de Persona, Familia y Relaciones Humanas tiende a regular (1.95), en la asignatura de Ciencia, Tecnología y Ambiente es regular (2.06), en la asignatura de Educación para el Trabajo es regular (2.11), el rendimiento académico en estas asignaturas es muy heterogéneo (desviación estándar promedio $=1,05$ ).

\section{DISCUSIÓN}

Las alteraciones de la afectividad en adolescentes se manifiestan con fatiga y falta de interés en los estudios, lentitud de pensamiento y palabra e intranquilidad, estos problemas afectan de 
modo grave al $1.2 \%$ de los estudiantes; de modo moderado al $15.3 \%$ y se presentan ligeramente en el $41.2 \%$; lo que indicaría que el $57.7 \%$ son de algún modo afectados por estos problemas.

Estos problemas han sido catalogados por múltiples autores como manifestaciones de la depresión en adolescentes, además se debemos de comprender que el ser humano necesita 'motivos' para actuar, deseos, ilusiones, objetivos que den sentido a su vida; a la depresión en adolescentes se llega, y una vez llegados, permanecemos en ella por una apatía, una desgana, una desilusión y falta de sentido en nuestras vidas que nos parece 'vegetativa', absurda.

Las alteraciones del estado anímico en adolescentesse manifiestan con Humor deprimido (tristeza, depresión en adolescentes, desamparo, inutilidad), Sensación de culpabilidad, Idea de suicidio, Depresión psíquica (irritabilidad) e Hipocondría, estos problemas afectan de modo grave al $1.2 \%$ de los estudiantes; de modo moderado al $2.4 \%$ y se presentan ligeramente en el $38.8 \%$; lo que indicaría que el $42.3 \%$ son de algún modo afectados por estos problemas.

Los estados de ánimo depresivos se pueden centrar en ciertas características de su organización psíquica que atañen, por un lado al Yo, y por otro, al objeto, objeto cuya pérdida no es compatible con el duelo normal.

Los problema físicos en adolescentes se manifiestan con Síntomas gastrointestinales: boca seca, flatulencia, diarrea, eructos, retortijones, Síntomas cardiovasculares: palpitaciones, cefalalgias, Síntomas genitales como pérdida de la libido (trastornos menstruales) y Pérdida de peso, estos problemas afectan de modo grave al $1.2 \%$ de los estudiantes; de modo moderado al $4.7 \%$ y se presentan ligeramente en el $56.5 \%$; lo que indicaría que el $62.4 \%$ son de algún modo afectados por estos problemas

El adolescente depresivo presenta una amplia variedad de síntomas que pueden ser clasificados como corporales, emocionales, y anímicos. Los síntomas corporales que más predominan incluyen dolores corporales crónicos, dolores de cabeza crónicos, problemas para dormir (insomnio severo), problemas con despertarse temprano y para comer (cambios de apetito, anorexia, rápida pérdida de peso); también predomina el dolor de cabeza crónico, y la disminución del deseo sexual - desde impotencia en los hombres hasta amenorrea (ausencia de la menstruación) o frigidez en las mujeres. Los síntomas emocionales por lo general son melancolía, depresión, y preocupación sobre el pasado, presente, y futuro. Finalmente, los síntomas anímicos consisten en pensamientos suicidas, y deseos de morir, y declaraciones como "la mañana es la peor parte del día". El dolor de cabeza que cohabita con la depresión en adolescentes es considerado un dolor de cabeza tensional (tensión muscular).

Los problemas en los procesos cognitivos en adolescentes se manifiestan con pérdida de la capacidad memorística, problemas para mantener la atención y concentración, problemas para poder comprender las cosas, sensación de cansancio mental y aburrimiento, falta de claridad de pensamiento y sensación de abrumamiento, estos problemas afectan de modo grave al 7.1 $\%$ de los estudiantes; de modo moderado al 5.9 $\%$ y se presentan ligeramente en el $60 \%$; lo que indicaría que el $72.9 \%$ son de algún modo afectados por estos problemas.

Con frecuencia, la depresión desvirtúa la percepción que el adolescente tiene de su propia memoria, lo que le conduce a consultar al médico, ya que tiene la impresión de que sus facultades para memorizar están decayendo. Generalmente, al adolescente le resulta complicado describir sus problemas con precisión. El estado depresivo entraña una disminución de la capacidad de concentración y en consecuencia, de memorización. En términos generales, cuando 
se tiene baja la moral o se sufre depresión, de una manera u otra, la memoria se ve afectada.

En relación al rendimiento académico se ha encontrado que en el $28.2 \%$ es bueno, en el $42.4 \%$ es regular, en el $23.5 \%$ es malo y en el 2.4. \% es deficiente. La depresión en el escolar, uno de los síntomas más comunes de la tensión emocional, es una importante pero desatendida área de investigación. Cada vez más escolares presentan problemas emocionales en la escuela. Estudios epidemiológicos indican que los desórdenes de depresión durante el desarrollo afectan aproximadamente al $9 \%$ de los niños (Berstein y Borchardt 1991) y entre el 13 y el 17\% de los jóvenes (Kashani y Orvaschel 1990). Otras referencias indican que entre el 10 y el $20 \%$ de los escolares se ven afectados por problemas relacionados con la depresión (Barret 1998).

Se puede observar que el coeficiente de correlación de Pearson es moderada negativa $-600^{* *}$ con un nivel de significancia de 0,000 , lo que significa que cuando los problemas afectivos (reflejados en modificaciones de la conducta) se incrementan disminuye el rendimiento académico, y esa afirmación puede hacerse con el $0.0 \%$ de probabilidad se equivocarnos (Tabla 01).

Tabla № 1.- Correlación entre estado afectivo (reflejado en la conducta) y el rendimiento académico en los estudiantes de la institución educativa mariscal castilla de el Tambo Huancayo en el 2009.

Además el coeficiente de determinación que es el coeficiente de correlación al cuadrado sale 0.36 lo que significa que el rendimiento académico es explicado en un $36 \%$ por el estado afectivo (reflejados en modificaciones de la conducta) del adolescente.

Se puede observar que el coeficiente de correlación de Pearson es débil negativa, $475^{\star *}$ con un nivel de significancia de 0,000, lo que significa que cuando los problemas afectivos (reflejados en los estados anímicos) se incrementan disminuye el rendimiento académico, y esa afirmación puede hacerse con el $0.0 \%$ de probabilidad se equivocarnos (Tabla $02)$.

Tabla № 2.- Correlación entre estado afectivo (reflejado en los estados anímicos) y el rendimiento académico en los estudiantes de la institución educativa mariscal castilla de El Tambo - Huancayo en el 2009.

\begin{tabular}{|c|c|c|c|}
\hline & CORRELACIONES & $\begin{array}{r}\text { Rendimiento } \\
\text { académico }\end{array}$ & $\begin{array}{l}\text { ESTADO } \\
\text { ANIMICO }\end{array}$ \\
\hline $\begin{array}{l}\text { RENDIMIENTO } \\
\text { ACADÉMICO }\end{array}$ & $\begin{array}{l}\text { Correlación de Pearson } \\
\text { Sig. (bilateral) } \\
N\end{array}$ & $\begin{array}{l}1 \\
85\end{array}$ & $\begin{array}{l}-, 475^{* \star} \\
, 000 \\
85\end{array}$ \\
\hline $\begin{array}{r}\text { ESTADO ANÍMICO } \\
* * \text { La correl }\end{array}$ & $\begin{array}{l}\text { Correlación de Pearson } \\
\text { Sig. (bilateral) } \\
N \\
\text { ión es significativa al nive }\end{array}$ & $\begin{array}{c}-, 475^{\star \star} \\
, 000 \\
85 \\
\text { el } 0,01 \text { (bilatera }\end{array}$ & $\begin{array}{l}1 \\
85\end{array}$ \\
\hline
\end{tabular}

Además el coeficiente de determinación que es el coeficiente de correlación al cuadrado sale 0.225 lo que significa que el rendimiento académico es explicado en un $22.5 \%$ por el estado afectivo (reflejados en los estados anímicos) del adolescente.

Se puede observar que el coeficiente de correlación de Pearson es moderada negativa ,$- 651^{* *}$ con un nivel de significancia de 0,000 , lo que significa que cuando los problemas afectivos (reflejados en el estado físico) se incrementan disminuye el rendimiento académico, y esa afirmación puede hacerse con el $0.0 \%$ de probabilidad se equivocarnos (Tabla 03)

Tabla $N^{0}$ 3.- Correlación entre estado afectivo (reflejado en el estado físico) y el rendimiento académico en los estudiantes de la institución educativa mariscal castilla de El Tambo - 
Huancayo en el 2009.

\begin{tabular}{|c|c|c|c|}
\hline \multicolumn{4}{|c|}{ CORRELACIONES } \\
\hline & & RENDIMIENTO & ESTADO \\
\hline & & ACADÉMICO & FÍSICO \\
\hline \multirow{4}{*}{$\begin{array}{l}\text { RENDIMIENTO } \\
\text { ACADÉMICO }\end{array}$} & Correlación de & 1 & $-651^{* k}$ \\
\hline & Pearson & & \\
\hline & Sig. (bilateral) & &, 000 \\
\hline & N & 85 & 85 \\
\hline \multirow[t]{4}{*}{ ESTADO FÍSICO } & Correlación de & $-651^{* *}$ & 1 \\
\hline & Pearson & & \\
\hline & Sig. (bilateral) &, 000 & \\
\hline & N & 85 & 85 \\
\hline \multicolumn{4}{|c|}{ **. La correlación es significativa al nivel 0,01 (bilateral). } \\
\hline
\end{tabular}

Además el coeficiente de determinación que es el coeficiente de correlación al cuadrado sale 0.42 lo que significa que el rendimiento académico es explicado en un $42 \%$ por el estado afectivo (reflejados en el estado físico) del adolescente.

Se puede observar que el coeficiente de correlación de Pearson es moderada negativa ,$- 644^{* *}$ con un nivel de significancia de 0,000 , lo que significa que cuando los problemas afectivos (reflejado en los procesos cognitivos) se incrementan disminuye el rendimiento académico, y esa afirmación puede hacerse con el $0.0 \%$ de probabilidad se equivocarnos (Tabla 04)

Tabla N ${ }^{0}$ 4.- Correlación entre estado afectivo (reflejado en los procesos cognitivos) y el rendimiento académico en los estudiantes de la institución educativa mariscal castilla de El Tambo - Huancayo en el 2009.

\begin{tabular}{|c|c|c|c|}
\hline \multicolumn{4}{|c|}{$\begin{array}{l}\text { CORRELACIONES } \\
\end{array}$} \\
\hline & & $\begin{array}{l}\text { RENDIMIENTO } \\
\text { ACADÉMICO }\end{array}$ & $\begin{array}{l}\text { PROBLEMAS } \\
\text { COGNITIVOS }\end{array}$ \\
\hline $\begin{array}{l}\text { RENDIMIENTO } \\
\text { ACADÉMICO }\end{array}$ & $\begin{array}{l}\text { Correlación de } \\
\text { Pearson }\end{array}$ & 1 & $-644^{* *}$ \\
\hline & $\begin{array}{c}\text { Sig. (bilateral) } \\
\mathrm{N}\end{array}$ & 85 & $\begin{array}{l}, 000 \\
85\end{array}$ \\
\hline $\begin{array}{l}\text { PROBLEMAS } \\
\text { COGNITIVOS }\end{array}$ & $\begin{array}{l}\text { Correlación de } \\
\text { Pearson }\end{array}$ & ; $644^{* \star}$ & 1 \\
\hline & $\begin{array}{l}\text { Sig. (bilateral) } \\
N \\
\text { relación es signif }\end{array}$ & $\begin{array}{c}, 000 \\
85 \\
\text { tiva al nivel } 0 \text {, }\end{array}$ & $\begin{array}{l}85 \\
\text { ateral). }\end{array}$ \\
\hline
\end{tabular}

Además el coeficiente de determinación que es el coeficiente de correlación al cuadrado sale 0.414 lo que significa que el rendimiento académico es explicado en un $41 \%$ por el estado afectivo (reflejado en los procesos cognitivos) del adolescente.

La presentación de problemas de conducta, estado anímico, estado físico, y procesos cognitivos que denotan alteración del estado afectivo son ligeros y afecta de algún modo al $57.7 \%$ de los estudiantes del $4^{\circ}$ y $5^{\circ}$ año de la Institución Educativa Mariscal Castilla. El rendimiento académico en las asignaturas de Matemática, Comunicación, Arte, Inglés, Formación Ciudadana y Cívica, Historial Geografía y Economía, Persona/Familia y Relaciones Humanas, Ciencia/Tecnología y Ambiente y Educación para el Trabajo en promedio es regular en los estudiantes del $4^{\circ}$ y $5^{\circ}$ año de la Institución Educativa Mariscal Castilla.

Referente a la relación entre la afectividad (reflejado en la conducta) y el rendimiento académico, se puede observar que el coeficiente de correlación de Pearson es moderada negativa $\left(-0,600^{* \star}\right)$, lo que significa que cuando los problemas afectivos (reflejados en modificaciones de la conducta) se incrementan disminuye el rendimiento académico, y esa afirmación puede hacerse con el $0.0 \%$ de probabilidad se equivocarnos, con ello se prueba la hipótesis planteada que: A mayor modificación del estado afectivo (reflejado en la conducta) menor rendimiento académico en los estudiantes de la institución educativa Mariscal Castilla.

Referente a la relación entre la afectividad (reflejados en los estados anímicos) y el rendimiento académico, se puede observar que el coeficiente de correlación de Pearson es débil negativa $-0,475^{\star *}$, lo que significa que cuando los problemas afectivos (reflejados en los estados anímicos) se incrementan disminuye el rendimiento académico, y esa afirmación 
puede hacerse con el $0.0 \%$ de probabilidad se equivocarnos, con ello se prueba la hipótesis planteada que: A mayor modificación del estado afectivo (reflejados en los estados anímicos) menor rendimiento académico en los estudiantes de la institución educativa Mariscal Castilla.

Referente a la relación entre la afectividad (reflejados en el estado físico) y el rendimiento académico, se puede observar que el coeficiente de correlación de Pearson es moderada negativa $-0,651^{* *}$, lo que significa que cuando los problemas afectivos (reflejados en el estado físico) se incrementan disminuye el rendimiento académico, y esa afirmación puede hacerse con el $0.0 \%$ de probabilidad se equivocarnos, con ello se prueba la hipótesis planteada que: A mayor modificación del estado afectivo (reflejados en el estado físico) menor rendimiento académico en los estudiantes de la institución educativa Mariscal Castilla.

Referente a la relación la afectividad (reflejado en los procesos cognitivos) y el rendimiento académico, se puede observar que el coeficiente de correlación de Pearson es moderada negativa $-0,644^{* *}$, lo que significa que cuando los problemas afectivos (reflejado en los procesos cognitivos) se incrementan disminuye el rendimiento académico, y esa afirmación puede hacerse con el $0.0 \%$ de probabilidad se equivocarnos, con ello se prueba la hipótesis planteada que: A mayor modificación del estado afectivo (reflejado en los procesos cognitivos) menor rendimiento académico en los estudiantes de la institución educativa Mariscal Castilla.

\section{REFERENCIAS BIBLIOGRÁFICAS}

Bandura, A: Teoría del aprendizaje social.
Espasa-Calpe, Madrid, 1984.

Bellack, L.; Siegel, H: Manual de psicoterapia breve, intensiva y de urgencia. Manual Moderno, México, 1986.

Gluhoski, V.Ly Young, J.E: El estado de la cuestión en la terapia cognitiva centrada en esquemas. En: Caro Gabalda, I: Manual de psicoterapias cognitivas, Paidós, Barcelona, 1997.

Greenberg, L.S; Rice, L.N; y Elliot, R: Facilitando el cambio emocional. Paidós, Barcelona, 1996.

Goldfried, M.R: De la terapia cognitiva-conductual a la psicoterapia de integración. D.D.B. Bilbao, 1995.

Lewinsohn, P.M, Gotlib, I.H y Hautzinger, M: Tratamiento conductual de la depresión en adolescentes. En-Caballo V.E: Manual para el tratamiento cognitivo-conductual de los trastornos psicológicos. Siglo XXI, Madrid, 1997.

Liberman, R.P: Rehabilitación integral del enfermo mental crónico. Martinez Roca, 1995.

Neimeyer, R.A y Mahoney, M: Constructivismo y psicoterapia. Paidós, Barcelona, 1998.

Ramirez-Basco, M y Thase, M: Tratamiento cognitivo-conductual de los trastornos bipolares. En-Caballo, V.E: Manual de tratamiento cognitivoconductual de los trastornos psicológicos. Siglo XXI, Barcelona, 1997.

Ribes, E: Psicología y salud: un análisis conceptual. Martinez Roca, Barcelona, 1990.

Ruiz, J.J: Fundamentos de psicoterapia cognitiva. 1Édición. A demanda, Jaén, 1994.

Safran, J.D y Segal, Z.V: El proceso interpersonal en la terapia cognitiva. Paidós, Barcelona, 1994. 